\title{
Lymph node density in papillary thyroid carcinoma is prognostic factor after adjusting for pathological stage
}

\author{
Hidenori Suzuki $^{1}$, Yusuke Koide ${ }^{1}$, Nobuhiro Hanai ${ }^{1}$, Daisuke Nishikawa ${ }^{1}$, Shintaro \\ Beppu $^{1}$, Shinji Mikami ${ }^{2}$ and Yasuhisa Hasegawa ${ }^{1}$ \\ ${ }^{1}$ Department of Head and Neck Surgery, Aichi Cancer Center Hospital, Nagoya, Japan \\ ${ }^{2}$ Department of Otolaryngology-Head and Neck Surgery, Nara Medical University, Kashihara, Japan \\ Correspondence to: Hidenori Suzuki, email: hi.suzuki@aichi-cc.jp \\ Keywords: lymph node density; papillary thyroid carcinoma; eighth edition of the Union for International Cancer Control TNM \\ Classification of Malignant Tumors; survival; multivariate \\ Received: March 14, $2018 \quad$ Accepted: April 24, $2018 \quad$ Published: June 01, 2018 \\ Copyright: Suzuki et al. This is an open-access article distributed under the terms of the Creative Commons Attribution License 3.0 \\ (CC BY 3.0), which permits unrestricted use, distribution, and reproduction in any medium, provided the original author and source \\ are credited.
}

\section{ABSTRACT}

We investigated the possible association between the lymph node density and survival outcomes in differentiated papillary thyroid carcinoma, and examined whether the Iymph node density was a predictor in a multivariate analysis adjusted for the pathological stage in the eighth edition of the Union for International Cancer Control Tumor-Node Metastasis Classification of Malignant Tumors. A total of 543 patients with papillary thyroid carcinoma were enrolled. We performed restaging according to the eighth edition. The lymph node density was the ratio between number of positive lymph nodes and total number of excised lymph nodes. A log-rank test and Cox's proportional hazards model were used for univariate and multivariate analysis with adjustment for the pathological stage in the eighth edition, respectively. In both the univariate and multivariate analyses of 150 patients with pN1bM0, the presence of a lymph node density of $\geq 0.3$ with $\mathrm{pN1b}$ was significantly associated with shorter disease-specific survival. In both the univariate and multivariate analyses of all 543 patients, a lymph node density of $\geq 0.3$ with pN1b were also significantly associated with shorter overall and disease-specific survival. In conclusion, these results suggest that the lymph node density can be used as a predictor for the survival outcomes after adjustment for the pathological stage in the eighth edition.

\section{INTRODUCTION}

Staging based on the tumor-node metastasis (TNM) classification is widely used as a prognostic factor for the survival outcomes, such as overall survival (OS) and disease-specific survival (DSS), in various types of carcinoma, including differentiated papillary thyroid carcinoma (PTC) [1]. The pathological stage (pStage) in the eighth edition of Union for International Cancer Control TNM classification of Malignant Tumors (UICC8th) was expected to be a better prognostic factor for many types of carcinoma, and significant associations were found between the survival outcomes and the stage when restaging based on the UICC8th was performed for oropharyngeal carcinoma and PTC [1-4]. However, it was difficult to predict OS and DSS using the same TNM stage $[5,6]$.

The lymph node density (LND), which is defined as the ratio of the number of positive lymph nodes to the number of total lymph nodes, has been shown to be associated with the survival outcomes in many types of carcinoma, including head and neck carcinoma [1, 5-9]. In a review and several articles of PTC, the LND was also shown to be a significant prognostic factor for the survival outcomes [1,7-9]. Recently, Amit et al. reported in a significant association between the LND and DSS in PTC based on a multivariate analysis with adjustment for clinicopathological factors, including the pathological $\mathrm{T}$ classification and $\mathrm{N}$ classification in the UICC8th; however, the analysis was not adjusted for the pathological stage in the UICC8th or the LND [1]. To the best of our 
Table 1: Clinicopathological factors in 543 patients with papillary thyroid carcinoma

\begin{tabular}{|c|c|c|c|}
\hline Factor & & & \\
\hline Gender & & Female & $143 / 400$ \\
\hline Age & & $<55 / 55 \geq$ & $126 / 97 / 320$ \\
\hline $\begin{array}{l}\text { Pathological stage } \\
\text { (UICC7th) }\end{array}$ & $\begin{array}{c}\text { pStage I } \\
\text { pStage II } \\
\text { pStage III } \\
\text { pStage IVA } \\
\text { pStage IVB } \\
\text { pStage IVC }\end{array}$ & $\begin{array}{c}<45 \text { pTAnyNAnyM0 } \\
45 \geq \text { pT1 N0-X M0 } \\
<45 \text { pTAnyNAnyM1 } \\
45 \geq \text { pT2 N0N-X M0 } \\
45 \geq \text { pT3 N0-X M0 } \\
45 \geq \text { pT1-3 N1a M0 } \\
45 \geq \text { pT1-3 N1b M0 } \\
45 \geq \text { pT4a N0-1 M0 } \\
45 \geq \text { pT4b N0-1 M0 } \\
45 \geq \text { AnyT AnyN pM1 }\end{array}$ & $\begin{array}{c}123 \\
79 \\
3 \\
6 \\
58 \\
138 \\
97 \\
25 \\
0 \\
14\end{array}$ \\
\hline $\begin{array}{l}\text { Pathological stage } \\
\text { (UICC8th) }\end{array}$ & $\begin{array}{c}\text { pStage I } \\
\text { pStage II } \\
\text { pStage III } \\
\text { pStage IVA } \\
\text { pStage IVB }\end{array}$ & $\begin{array}{c}<55 \text { pTAnyNAnyM0 } \\
55 \geq \text { pT1-2 N0-X M0 } \\
<55 \text { pTAnyNAnyM1 } \\
55 \geq \text { pT3 N0-X M0 } \\
55 \geq \text { pT1-3 N1 M0 } \\
55 \geq \text { pT4a AnyN M0 } \\
55 \geq \text { pT4b AnyN M0 } \\
55 \geq \text { AnyT AnyN pM1 }\end{array}$ & $\begin{array}{c}220 \\
86 \\
3 \\
23 \\
173 \\
24 \\
0 \\
14\end{array}$ \\
\hline Positive surgical margin & & /Absence & $70 / 473$ \\
\hline Operation & Total & ectomy/Others & $251 / 292$ \\
\hline Adjuvant RAI & & /Absence & $59 / 484$ \\
\hline Charlson comorbidity index & & $1 \geq$ & $272 / 271$ \\
\hline Survival outcome & Cancer-spe & th/ Other death/Alive & $20 / 23 / 500$ \\
\hline
\end{tabular}

Abbreviations: pStage: pathological stage; UICC8th: the eighth edition of the Union for International Cancer Control TNM Classification of Malignant Tumors; UICC7th: the seventh edition of the Union for International Cancer Control TNM Classification of Malignant Tumors; RAI: radioactive iodine.

knowledge, no studies have investigated whether the LND is a prognostic factor in PTC based on a multivariate analysis with adjustment for the pathological stage in the UICC8th.

In the present study, we investigated the possible association between the LND and the survival outcomes of patients with PTC, and examined whether the LND was a prognostic factor in a multivariate analysis with adjustment for the pathological stage in the UICC8th.

\section{RESULTS}

\section{Clinicopathological factors and pStage}

The clinicopathological factors were shown in Table 1. The association between the pStage in the UICC7th and the pStage in the UICC8th is shown in Table 2. Of the 306 patients with pStage I in the UICC8th, 202, 6, 74, and 24 patients were restaged from pStage I, II, III, and IVA in the UICC7th, respectively. Of the 199 patients with pStage II in the UICC8th, 3, 122, and 74 patient were restaged from pStage II, III, and IVA in the UICC7th, respectively.
Twenty-four patients with pStage III and 14 patients with pStage IVB in the UICC8th were restaged from 24 patients with pStage IVA and 14 patients with pStage IVC in the UICC7th, respectively.

\section{Clinical course}

At the end of the study, the mean $\pm \mathrm{SD}$ follow-up period among the whole population, the 500 surviving patients $(92.1 \%$, vs all), the 43 patients who died $(7.9 \%)$ and the 20 patients $(3.7 \%$, vs all) who died due to PTC was $2205 \pm 1284,2234 \pm 1298,1875 \pm 1073,1616 \pm$ 1032 days, respectively. In the study population, the 3-, 5-, 10-years OS rates were $97.5 \%, 94.6 \%$, and $84.5 \%$, respectively. While the 3-, 5-, 10-years DSS rates were $98.3 \%, 97.0 \%$, and $93.6 \%$, respectively. The causes of death for 20 patients who died due to PTC were distant failure $(n=15)$, locoreginal relapse $(n=4)$, and pulmonary embolism as a complication $(n=1)$. The causes of death for 23 patients who died due reasons to other than PTC were other malignancy $(n=14)$, unknown $(n=8)$, and interstitial pneumonia $(n=1)$. 
Table 2: Association between the pStages in the UICC7th and UICC8th

\begin{tabular}{|c|c|c|c|c|c|}
\hline & \multicolumn{5}{|c|}{ UICC 7th } \\
\hline & $\begin{array}{c}\text { pStageI } \\
(n=202)\end{array}$ & $\begin{array}{c}\text { pStageII } \\
(n=9)\end{array}$ & $\begin{array}{c}\text { pStageIII } \\
(n=196)\end{array}$ & $\begin{array}{c}\text { pStageIVA } \\
(n=122)\end{array}$ & $\begin{array}{c}\text { pStageIVC } \\
(n=14)\end{array}$ \\
\hline UICC8th & & & & & \\
\hline pStageI $\quad(n=306)$ & 202 & 6 & 74 & 24 & 0 \\
\hline pStageII $\quad(n=199)$ & 0 & 3 & 122 & 74 & 0 \\
\hline pStageIII $(n=24)$ & 0 & 0 & 0 & 24 & 0 \\
\hline pStageIVB $(n=14)$ & 0 & 0 & 0 & 0 & 14 \\
\hline
\end{tabular}

Abbreviations: pStage: pathological stage; UICC8th: the eighth edition of the Union for International Cancer Control TNM Classification of Malignant Tumors; UICC7th: the seventh edition of the Union for International Cancer Control TNM Classification of Malignant Tumors.

\section{Survival analysis based on pStage on the UICC7th and the UICC8th in all 543 patients}

In the univariate analysis of all 543 paients, both the pStage in the UICC7th among the 5 staging groups (pStageI, II, III, IVA, IVC) $(p<0.0001)$ and pStage in the UICC8th among the 4 staging groups (pStageI, II, III, IVB) $(p<0.0001)$ were significantly associated with OS after the Bonferroni's correction. The Kaplan-Meier curves from the univariate analyses of OS for the pStage in the UICC 7th and the pStage in the UICC8th are shown in Figure 1. In the multivariate analysis adjusted for the pStage in the UICC7th and the pStage in the UICC8th (Table 3), the pStage in the UICC8th was significantly associated with OS after the Bonferroni's correction. (per pStage: HR 2.22, 95\% CI 1.38-3.70, $p=0.0008$ )

\section{Survival analysis based on LND in 150 patients with pN1bM0}

In 150 patients with $\mathrm{pN} 1 \mathrm{bM} 0$, the pStage in the UICC8th (I/II/III) was significantly associated with DSS $(p<0.0001)$ after Bonferroni's correction. The median and mean \pm SD LND of the 150 patients with pN1bM0 was 0.3 and $0.32 \pm 0.17$, respectively (Figure 2 ). We divided the patients into 2 groups based on the median LND (0.3). Among the pN1bM0 patients, LND $\geq 0.3(n=75)$ were significantly associated with a shorter DSS in comparison to LND $<0.3(n=75)(p=0.0090)$; non-significance with Bonferroni's correction of the significance level $p<$ 0.0042. The Kaplan-Meier curves from the univariate OS analyses of both pStage in the UICC8th and LND (LND $\geq 0.3 / \mathrm{LND}<0.3$ ) for the 150 patients with $\mathrm{pN} 1 \mathrm{bM} 0$ are

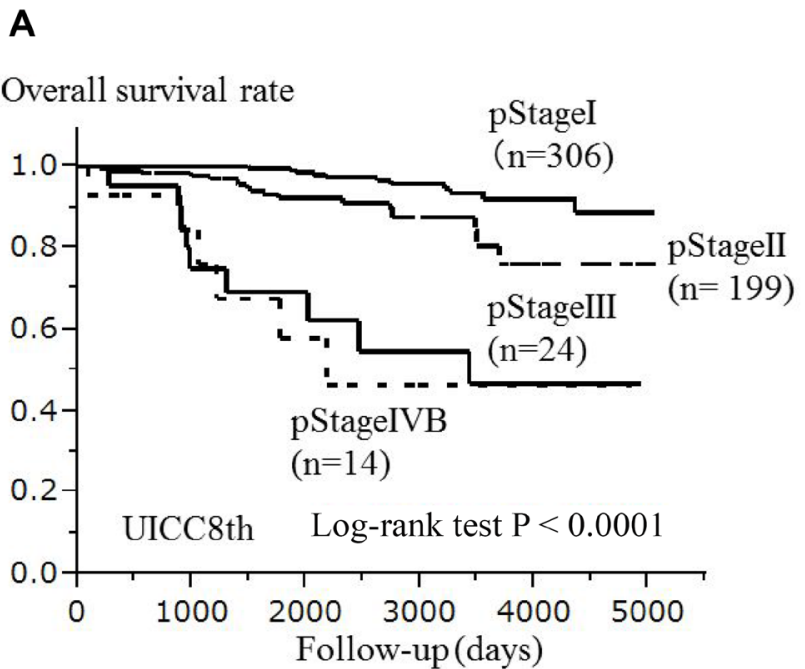

B

Overall survival rate

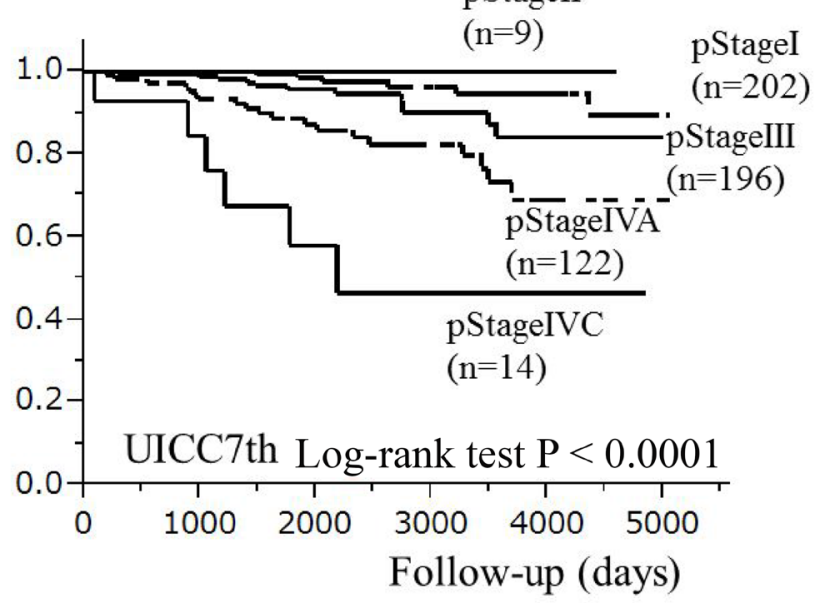

Figure 1: The association between the pathological stage and the overall survival of 543 patients with papillary thyroid carcinoma (Kaplan-Meier method). The pStages in the UICC8th $(p<0.0001)$ (A) and UICC7th $(p<0.0001)$ (B) were significantly associated with overall survival after the Bonferroni's correction (log-rank test). Abbreviations: pStage: pathological stage; UICC8th: eighth edition of the Union for International Cancer Control TNM Classification of Malignant Tumors; UICC7th: seventh edition of the Union for International Cancer Control TNM Classification of Malignant Tumors. 
Table 3: The multivariate analysis for adjustment with pStages in the UICC7th and UICC8th

\begin{tabular}{lccc}
\hline \multirow{2}{*}{ Factor } & \multicolumn{3}{c}{ Overall survival } \\
\cline { 2 - 4 } & Hazards ratio & $\mathbf{9 5 \%}$ Confidence interval & $\boldsymbol{P}$-value \\
\hline pStage on UICC8th & 2.22 & $1.38-3.70$ & 0.0008 \\
pStage on UICC7th & 1.27 & $0.83-1.95$ & 0.26 \\
\hline
\end{tabular}

Abbreviations: pStage: pathological stage; UICC8th: the eighth edition of the Union for International Cancer Control TNM Classification of Malignant Tumors; UICC7th: the seventh edition of the Union for International Cancer Control TNM Classification of Malignant Tumors.

shown in Figure 3. In the multivariate analysis adjusted for pStage in the UICC8th and LND in the 150 patients with pN1bM0, pStage in the UICC8th (per pStage: HR 4.18, $95 \%$ CI $1.61-11.8, p=0.0033)$ and the presence of LND $\geq 0.3$ with pN1bM0 (HR 17.5, 95\% CI 2.58-138.4, $p=$ 0.0013 ) was significantly associated with the DSS after the Bonferroni's correction. A multivariate analysis of the DSS in the 150 patients with pN1bM0 is shown in Table 4.

\section{Univariate survival analysis based on LND in all 543 patients}

Among the 15 patients with pN1bM1, 9 patients had LND $\geq 0.3$ with $\mathrm{pN} 1 \mathrm{~b}$. In total, 84 patients had LND $\geq 0.3$ with pN1b, this included 75 patients with pN1bM0 and 9 patients with $\mathrm{pN} 1 \mathrm{bM} 1$. In all 543 patients, the presence of LND $\geq 0.3$ with $\mathrm{pN1b}(n=84)$ was significantly associated with shorter OS $(p<0.0001)$ and DSS $(p$ $<0.0001$ ) in comparison to the absence of LND $\geq 0.3$ with pN1b $(n=459)$ after the Bonferroni's correction. The Kaplan-Meier curves from both OS and DSS of the presence/absence of LND $\geq 0.3$ with $\mathrm{pN} 1 \mathrm{~b}$ are shown in Figure 4.

\section{Multivariate survival analysis in all 543 patients}

The results of the multivariate analyses of both OS and DSS in all 543 patients are shown in Table 5. In the multivariate analysis with adjustments for pStage in the UICC8th and LND $\geq 0.3$ with pN1b, pStage in the UICC8th was significantly associated with both OS (per pStage: HR 2.40, 95\% CI 1.76-3.24, $p<0.0001$ ) and DSS (per pStage: HR 2.53, 95\% CI 1.67-3.85, $p<0.0001$ ) after the Bonferroni's correction, the presence of LND $\geq 0.3$ with $\mathrm{pN} 1 \mathrm{~b}$ was significantly associated with the OS (HR $2.50,95 \%$ CI 1.27-4.82, $p=0.0086$ ); non-significance with the Bonferroni's correction of the significance level $p<0.0042$, and the presence of LND $\geq 0.3$ with $\mathrm{pN} 1 \mathrm{~b}$

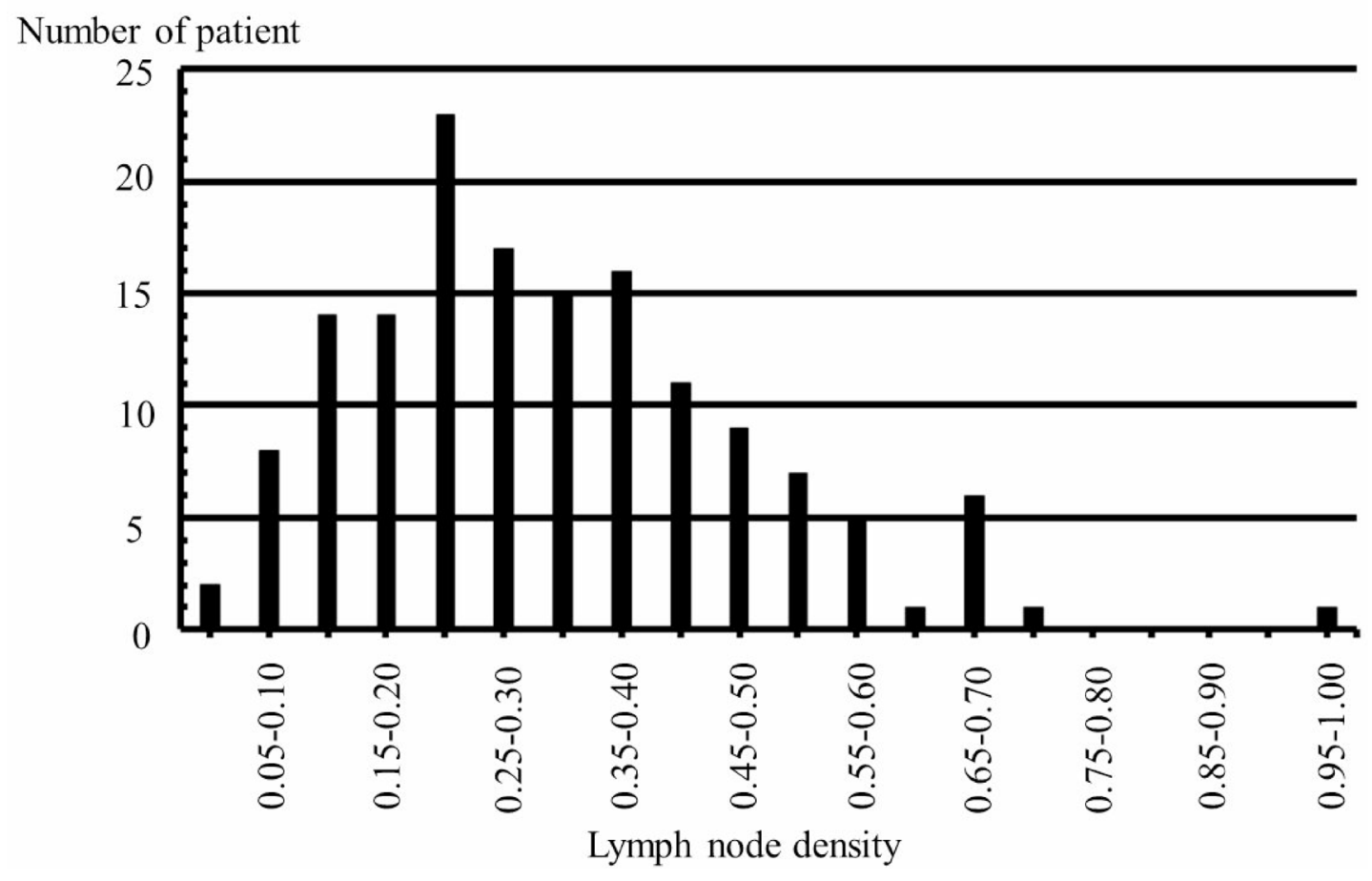

Figure 2: Association between the lymph node density and number of patients with pN1bM0 in 150 papillary thyroid carcinoma patients. 
was significantly associated with both the DSS (HR 9.78, 95\% CI 3.49-31.7, $p<0.0001)$ after the Bonferroni's correction. In the multivariate analysis after adjusting for the pStage in the UICC8th, LND $\geq 0.3$ with $\mathrm{pN} 1 \mathrm{~b}$, gender, positive surgical margin, thyroidectomy, adjuvant RAI, and CCI, pStage in the UICC8th (per pStage: HR 2.33, 95\% CI $1.66-3.24, p<0.0001)$ was significantly associated with shorter OS after the Bonferroni correction, the presence of LND $\geq 0.3$ with pN1b (HR 2.22, 95\% CI 1.08-4.52, $p=0.0305)$ was significantly associated with shorter OS; non-significance with the Bonferroni's correction of the significance level $p<0.0042$, and the pStage in the UICC8th (per pStage: HR 2.17, 95\% CI 1.38-3.46, $p=$ 0.0009 ), presence of LND $\geq 0.3$ with $\mathrm{pN} 1 \mathrm{~b}$ (HR 6.04, 95\% CI 2.08-20.6, $p=0.0007$ ), male gender (HR 3.55, 95\% CI $1.27-10.4, p=0.00154$ ) were associated with shorter DSS significantly after the Bonferroni's correction.

\section{DISCUSSION}

In the present study, we showed for the first time in both univariate and multivariate analyses adjusted for the pStage in the UICC8th that PTC patients of LND $\geq 0.3$ with $\mathrm{pN} 1 \mathrm{bM} 0$ had significantly shorter DSS, and that patients with LND $\geq 0.3$ with $\mathrm{pN} 1 \mathrm{~b}$ had significantly shorter OS and DSS.

Restaging of the pStage on UICC 8th from the pStage of the seventh edition according to the eighth edition has been reported to be superior for predicting survival in various types of carcinoma, including PTC [2-4]. Pontius et al. reported in PTC that the differences (UICC7th vs UICC8th) were age for cut point (45 vs 55 ), extrathyroidal extension (minimal vs gross), and $\mathrm{N}$ stage (N1a and N1b vs single N1), and that the UICC8th model showed a better fit to the data in comparison to the UICC7th model [2]. The findings of the present study showed that the pStage in the UICC8th was significantly associated with OS in a multivariate analysis adjusted for the pStage in the UICC7th, which is in agreement with the findings of previous studies [2-4].

The LND, which is influenced by nodal staging, surgical, and pathological factors, has been shown to be a prognostic factor in many types of carcinoma, regardless of the types of neck dissection that are used [5]. Because the LND was reported to be affected by the number of lymph nodes retrieved based on the extent of surgery [7], we analyzed about patients with N1b rather than $\mathrm{N} 1$, despite on the UICC8th. LND value may change depending on the criteria to submit lymph nodes for a pathological examination. We also reported that there was a significant association between LND and the survival outcomes including the OS and DSS in hypopharyngeal and oral squamous cell carcinoma $[5,6]$. Lee et al. reported that a higher LND was significantly associated with shorter survival in PTC with pN1b [8]. Moreover, a recent review for nine articles to investigate the prognostic value of the LND in PTC showed a significant association between the LND and the survival outcomes, and the authors noted that a lymph node ratio of $\geq 0.3$ was associated with an increased risk of recurrence [2-4]. The findings of the present study revealed significant associations between the LND and survival outcomes, and are in good agreement with the results of previous studies [5-8]. The cut-off values of continuous variable in a scientific study as systemic review and meta-analysis have been determined by the lowest $p$-value, receiver-operating
A

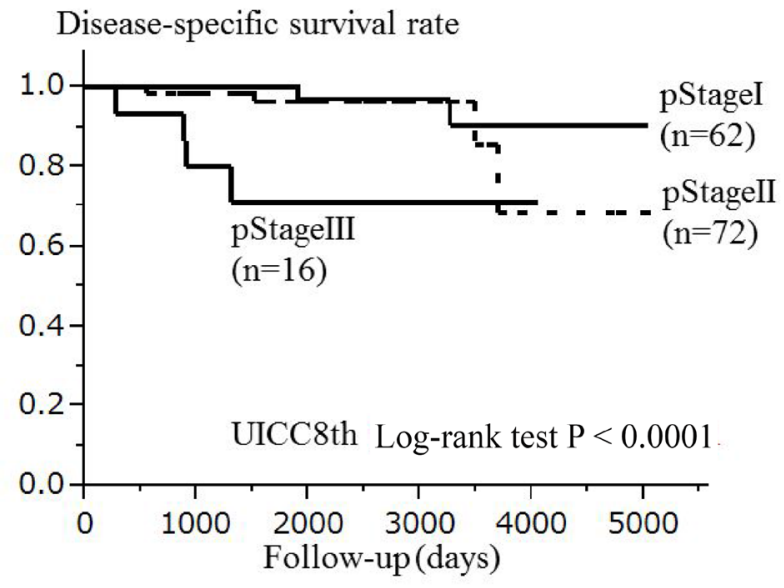

B

Disease-specific survival rate

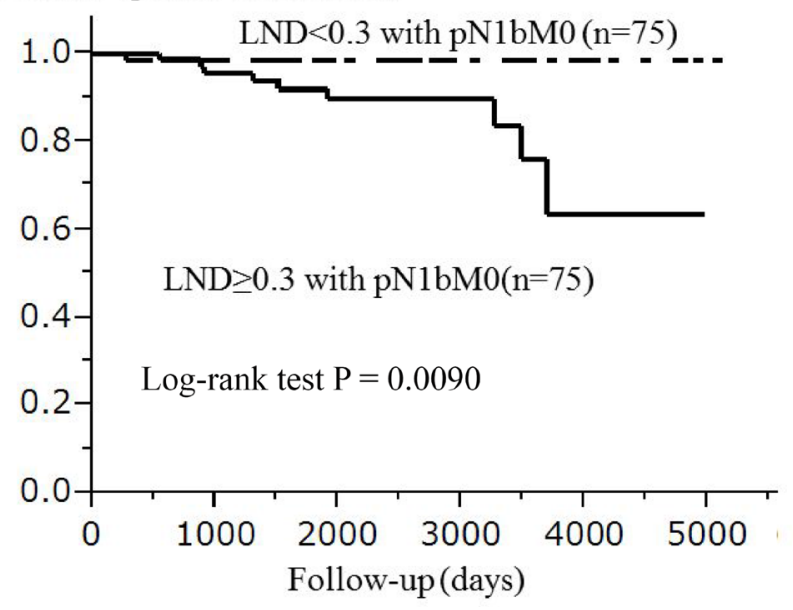

Figure 3: In 150 papillary thyroid carcinoma patients with pN1bM0 (Kaplan-Meier method), the pStage in the UICC8th $(p<0.0001)$ (A) was significantly associated with disease-specific survival after the Bonferroni's correction, and the lymph node density $(p=0.0090)$ (B) were significantly associated with disease-specific survival; non-significance with Bonferroni's correction of the significance level $p<0.0042$. The log-rank test was used for the statistical analysis. Abbreviations: pStage: pathological stage; UICC8th: eighth edition of the Union for International Cancer Control TNM Classification of Malignant Tumors; LND: lymph node density. 
Table 4: The multivariate disease-specific survival analysis for adjustment with the pStage in the UICC8th (I/II/III) and LND (LND $\geq 0.3$ with $\mathrm{pN} 1 \mathrm{bM0} / \mathrm{LND}<0.3$ with $\mathrm{pN1bM0)}$ in $\mathrm{pN1bM0}$

\begin{tabular}{lccc}
\hline \multirow{2}{*}{ Factor } & \multicolumn{3}{c}{ Disease-specific survival } \\
\cline { 2 - 4 } & HR & $\mathbf{9 5 \%}$ CI & $\boldsymbol{P}$-value \\
\hline $\begin{array}{l}\text { pStage in the UICC8th } \\
\text { (I/II/III) }\end{array}$ & 4.18 & $1.61-11.8$ & 0.0033 \\
$\begin{array}{l}\text { LND } \\
\text { (LND } \geq 0.3 \text { with pN1bM0/LND }<0.3 \text { with pN1bM0) }\end{array}$ & 17.5 & $2.58-138.4$ & 0.0013 \\
\hline
\end{tabular}

Abbreviations: pStage: pathological stage; HR: Hazards ratio; 95\% CI: 95\% confidence interval; UICC8th: the eighth edition of the Union for International Cancer Control TNM Classification of Malignant Tumors; LND: lymph node density.

characteristic, and a median value [14], so we selected the median value of LND (0.3) as the lucid determination in the present study.

Amit et al. reported that a higher LND was significant associated with shorter OS and DSS in univariate and multivariate analyses in 2542 patients with PTC, and that the multivariate analysis was adjusted for clinicopathological factors, including the pathological T and $\mathrm{N}$ classification in the UICC8th [1]. To the best of our knowledge, no studies have reported the results of a multivariate analysis adjusted for the LND and pStage in the UICC8th. Thus, we considered that there was a need for such an analysis. In the present study, in which we performed a multivariate analysis adjusted for the pStage in the UICC8th, we found a significant association between the LND and the survival outcomes of patients with in PTC. Because the stage based on both the T and $\mathrm{N}$ classification is comprehensive, we considered that a multivariate analysis adjusted for the pathological stage would be superior to the analyses made in the previous study by Amit et al, in which a multivariate analysis was performed with adjustment for the pathological $\mathrm{T}$ and $\mathrm{N}$ classification [1]. Based on the results of the present study, we have two plans to add adjuvant RAI and to monitor closely during follow up for patients with higher LND as described previously [7].

We believe that there was no possibility that the LND is confounded with any risk factors another than the clinical stage in UICC8th of PTC.

The present study was associated with some limitations. Specifically, it was retrospective in nature and the study population was relatively small. A future prospective study with a large cohort and multiinstitutional setting would yield more accurate and useful results. Because all lymph nodes were examined in a single representative cross section, as described previously [6], the present study could not detect the micro metastases of lymph nodes, which are well known to occur in cases of PTC. Because a lateral neck dissection generally is performed for the patients with lymph node metastasis detected at the lateral neck before surgery, we considered that a preoperative diagnosis might be a potential source of

\section{A}

Overall survival rate

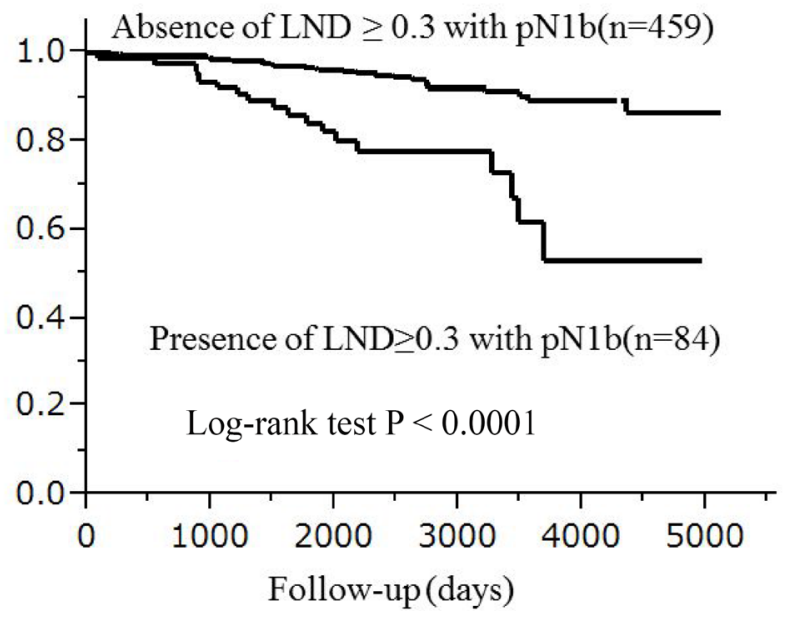

B

Disease-specific survival rate

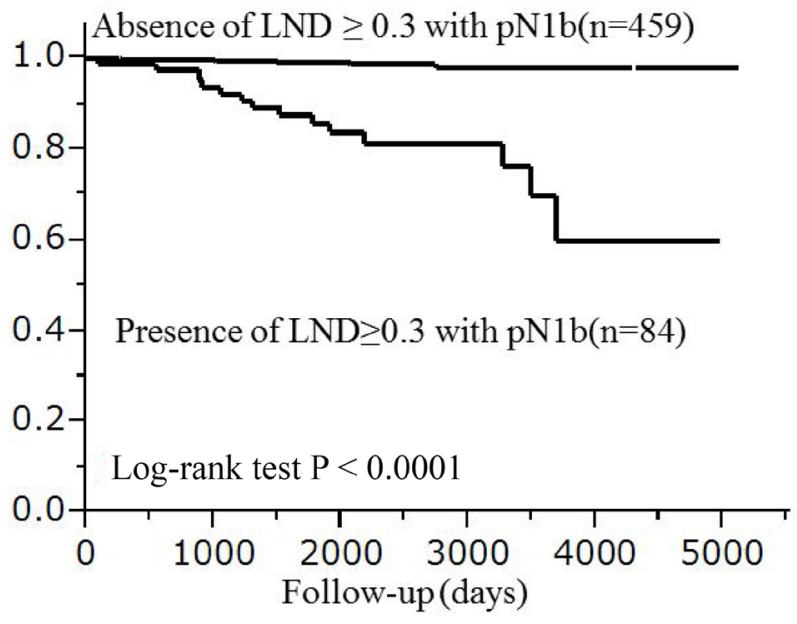

Figure 4: The association between LND and the survival of 543 patients with papillary thyroid carcinoma (KaplanMeier method). $\mathrm{LND} \geq 0.3$ was found to be associated with a significantly lower overall survival $(p<0.0001)(\mathbf{A})$ and disease-specific survival $(p<0.0001)$ (B) by log-rank test after the Bonferroni's correction. Abbreviation: LND: lymph node density. 
Table 5: The multivariate survival analysis in all patients $(n=543)$

\begin{tabular}{lcccccc}
\hline & \multicolumn{3}{c}{ Overall survival } & \multicolumn{3}{c}{ Disease-specific survival } \\
\cline { 2 - 7 } Factor & HR & $\mathbf{9 5 \% ~ C I ~}$ & $\boldsymbol{P}$-value & HR & $\mathbf{9 5 \% ~ C I ~}$ & $\boldsymbol{P}$-value \\
\hline Model 1 & & & & & & \\
$\quad$ pStage in the UICC8th (I/II/III/IVB) & 2.40 & $1.76-3.24$ & $<0.0001$ & 2.53 & $1.67-3.85$ & $<0.0001$ \\
LND $\geq 0.3$ with pN1b (Presence/Absence) & 2.50 & $1.27-4.82$ & 0.0086 & 9.78 & $3.49-31.7$ & $<0.0001$ \\
Model 2 & & & & & & \\
pStage in the UICC8th (I/II/III/IVB) & 2.33 & $1.66-3.24$ & $<0.0001$ & 2.17 & $1.38-3.46$ & 0.0009 \\
LND $\geq 0.3$ with pN1b (Presence/Absence) & 2.22 & $1.08-4.52$ & 0.0305 & 6.04 & $2.08-20.6$ & 0.0007 \\
Gender (Male/Female) & 1.42 & $0.71-2.76$ & 0.3191 & 3.55 & $1.27-10.4$ & 0.00154 \\
$\quad$ Positive surgical margin (Presence/Absence) & 2.02 & $0.89-4.14$ & 0.0902 & 2.76 & $0.91-7.54$ & 0.0708 \\
Thyroidectomy (Total /Others) & 1.32 & $0.64-2.76$ & 0.4491 & 2.39 & $0.64-11.5$ & 0.2041 \\
Adjuvant RAI (Presence/Absence) & 0.59 & $0.19-1.55$ & 0.3010 & 0.74 & $0.19-2.39$ & 0.6308 \\
CCI (CCI $\geq 1 / C C I=0)$ & 0.95 & $0.51-1.77$ & 0.8621 & 0.61 & $0.23-1.54$ & 0.2899 \\
\hline
\end{tabular}

Abbreviations: HR: Hazards ratio; 95\% CI: 95\% confidence interval; UICC8th: the eighth edition of the Union for International Cancer Control TNM Classification of Malignant Tumors; LND: lymph node density; RAI: radioactive iodine; CCI: Charlson comorbidity index.

bias. Because we analyzed not only patients with $\mathrm{pN} 1 \mathrm{bM} 0$ but also those with $\mathrm{pN} 1 \mathrm{~b}$, we considered that there was some risk of an increased $\alpha$-error in the present study.

In conclusion, the present study demonstrated, in both univariate and multivariate analysis adjusted for the pStage in the UICC8th, that the LND is significantly associated with shorter OS and DSS in patients with PTC. The LND can therefore be considered to be a prognostic factor that can predict the survival outcomes in patients with PTC.

\section{MATERIALS AND METHODS}

\section{Study design and patients}

Between 2003 January and 2016 August, 601 patients without a history of thyroid surgery underwent radical surgery for PTC at Aichi Cancer Center Hospital. We excluded 58 of these 601 patients who had the following pathological diagnoses: anaplastic carcinoma $(n=19)$, medullary carcinoma $(n=15)$, follicular carcinoma ( $n=13)$, poorly differentiated carcinoma $(n=7)$, carcinoma showing thymus-like differentiation $(n=3)$, squamous cell carcinoma $(n=1)$. Thus, 543 patients, who were pathologically diagnosed with PTC, were enrolled in this study. This study was approved by our institutional review board, and informed consent for the treatments and examinations was obtained from all of the patients.

\section{Clinical staging and surgery}

Clinical staging was determined from a routine physical examination, laryngoscopy, ultrasonography, enhanced cervical computed tomography, cytology and 18F-FDG-PET/CT as needed. The Charlson comorbidity index, which was a weighted index, was calculated based on 19 comorbid conditions. The TNM classification was determined based on the International Union Against Cancer classification. The 543 patients were classified into the total thyroidectomy group ( $n=$ $251)$, non-total thyroidectomy group $(n=292)$ according to the extent to their operation. For neck dissection, we performed en bloc dissection, as described by the Japan Neck Dissection Study Group [10]. We performed both lateral neck dissection and central neck dissection as preoperative staging for patients who were diagnosed with $\mathrm{cN} 1 \mathrm{~b}$ or intraoperative staging for patients who were diagnosed with $\mathrm{sN} 1 \mathrm{~b}$ based on the pathological diagnosis after palpation in the operation. We performed central neck dissection for patients who were diagnosed with carcinoma based on preoperative cytology or an intraoperative pathological examination.

\section{Follow-up after surgery}

After surgery, the patients were followed-up at our outpatient clinic by ultrasonography and thyroglobulin measurements with a surveillance schedule of approximately once every 6-12 months, and enhanced computed tomography or 18F-FDG-PET/CT as needed. Thyroid stimulating hormone suppression was performed after total thyroidectomy, and we made an effort to perform salvage surgery for locoregional recurrence when possible. Adjuvant radioactive iodine (RAI) was indicated for patients with distant metastasis from 2003 to 2009, and was recommended for high-risk patients with conditions such as distant metastasis and severe extrathyroid extension from 2010, based on the established guideline of the Japanese Society of Thyroid Surgeons and the 
Japanese Association of Endocrine Surgeons, as described previously [11].

\section{Pathological staging}

Neck dissection samples were separated according to the cervical region, and then the total number of lymph nodes was recorded. We submitted all lymph nodes obtained during neck dissection for a pathological examination. Pathological examinations of the lymph nodes were performed in a single representative cross section. All of the other pathological examination methods have been published previously [6]. The pathological diagnosis (pathological $\mathrm{T}$ and $\mathrm{N}$ classification, pStage, surgical margin) was made by two experienced pathologists, who completed each report. We determined the pathological TNM classification based on the pathological report according to the UICC7th. We redetermined the pStage based on the UICC8th using both the pathological report and the record of the intraoperative findings, as described by the eighth edition of the cancer staging manual of the American Joint Committee on Cancer and the UICC8th $[12,13]$.

\section{LND}

For the 150 patients $(27.6 \%)$ with $\mathrm{pN} 1 \mathrm{bM} 0$, a total of 6920 lymph nodes were evaluated; 2068 (29.9\%) were found to be pathologically positive. In these 150 patients, the mean \pm SD numbers of excised lymph nodes and positive lymph nodes were $46.1 \pm 29.3$ and $13.8 \pm 10.5$, respectively. In 17 patients with M1, 15 patients with cN1bM1 underwent lateral neck dissection; 2 patients with cN0-1aM1 did not undergo lateral neck dissection. For the 15 patients with pN1bM1, who underwent lateral neck dissection, a total of 748 lymph nodes were evaluated, and $274(36.6 \%)$ were found to be pathologically positive. In these 15 patients, the mean \pm SD numbers of excised lymph nodes and positive lymph nodes were $49.9 \pm 27.7$ and $18.3 \pm 12.2$, respectively. The LND was calculated using the following formula:

$\mathrm{LND}=$ the number of positive lymph nodes/the total number of excised lymph nodes.

\section{Statistical analysis}

Statistical analyses were performed using the JMP software package (version 9; SAS; Cary, NC, USA). Survival time as the period from surgery to survival endpoint was calculated by the Kaplan-Meier method. The survival endpoints were death for OS, and death from PTC for DSS. A univariate survival analysis was carried out using a log-rank test. A multivariate analysis was performed using Cox's hazards model; both the hazards ratio (HR) and $95 \%$ confidence interval $(95 \% \mathrm{CI})$ were assessed. In all 543 patients, univariate and multivariate analyses of OS according to the pStage in the UICC7th
(I/II/III/IVA/IVC) and UICC8th (I/II/III/IVB) were performed. One hundred fifty patients with $\mathrm{pN} 1 \mathrm{bM} 0$ were divided into 2 groups based on the median LND $(\mathrm{LND} \geq 0.3: n=75 ; \mathrm{LND}<0.3: n=75)$. Univariate and multivariate analyses of DSS according to the pStage in the UICC8th (I/II/III) and LND (LND $\geq 0.3$ with $\mathrm{pN} 1 \mathrm{bM} 0 / \mathrm{LND}<0.3$ with $\mathrm{pN} 1 \mathrm{bM} 0$ ) were performed.

Because 9 of 15 patients with pN1bM1 had the presence of LND $\geq 0.3$ with $\mathrm{pN} 1 \mathrm{~b}$, all 543 patients were divided into 2 groups according to the presence of LND $\geq 0.3$ with pN1b $(n=84)$ or the absence of LND $\geq 0.3$ with $\mathrm{pN} 1 \mathrm{~b}(n=459)$. We performed a univariate survival analysis compare both the OS and DSS according to the presence or absence of LND $\geq 0.3$ with $\mathrm{pN} 1 \mathrm{~b}$ in all 543 patients. We assessed two multivariate analysis models for both OS and DSS in all patients. One model was adjusted for the pStage in the UICC8th (I/II/III/ IVB) and LND $\geq 0.3$ with $\mathrm{pN} 1 \mathrm{~b}$ (presence/absence); the other model was adjusted with pStage in the UICC8th (I/ II/III/IVB), LND $\geq 0.3$ with $\mathrm{pN} 1 \mathrm{~b}$ (presence/absence), gender (male/female), CCI $(\mathrm{CCI} \geq 1 / \mathrm{CCI}=0)$, positive surgical margin (presence/absence), thyroidectomy (total/ others), adjuvant RAI (presence/absence). $P$ values of $<$ 0.05 were considered to indicate statistical significance. Given the risk of $\alpha$ - error from many statistical analyses, we additionally adjusted Bonferroni's correction for the $P$ values as reported [15]. $P$ values of $<0.0042$ were considered to indicate statistical significance after Bonferroni's correction, as 12 individual tests were performed $(0.05 / 12)$.

\section{CONFLICTS OF INTEREST}

The authors declare no conflicts of interest.

\section{GRANT SUPPORT}

This study was supported by JSPS KAKENHI Grant Number of 16K11253.

\section{REFERENCES}

1. Amit M, Tam S, Boonsripitayanon M, Cabanillas ME, Busaidy NL, Grubbs EG, Lai SY, Gross ND, Sturgis EM, Zafereo ME. Association of Lymph Node Density With Survival of Patients With Papillary Thyroid Cancer. JAMA Otolaryngol Head Neck Surg. 2018; 144:108-114.

2. Pontius LN, Oyekunle TO, Thomas SM, Stang MT, Scheri RP, Roman SA, Sosa JA. Projecting Survival in Papillary Thyroid Cancer: A Comparison of the Seventh and Eighth Editions of the American Joint Commision on Cancer/ Union for International Cancer Control Staging Systems in Two Contemporary National Patient Cohorts. Thyroid. 2017; 27:1408-1416. 
3. Suh S, Kim YH, Goh TS, Lee J, Jeong DC, Oh SO, Hong JC, Kim SJ, Kim IJ, Pak K. Outcome prediction with the revised American joint committee on cancer staging system and American thyroid association guidelines for thyroid cancer. Endocrine. 2017; 58:495-502.

4. Mizumachi T, Homma A, Sakashita T, Kano S, Hatakeyama $\mathrm{H}$, Fukuda S. Confirmation of the eighth edition of the AJCC/UICC TNM staging system for HPV-mediated oropharyngeal cancer in Japan. Int J Clin Oncol. 2017; 22:682-689.

5. Suzuki H, Matoba T, Hanai N, Nishikawa D, Fukuda Y, Koide Y, Hasegawa Y. Lymph node ratio predicts survival in hypopharyngeal cancer with positive lymph node metastasis. Eur Arch Otorhinolaryngol. 2016; 273:4595-4600.

6. Suzuki H, Beppu S, Hanai N, Hirakawa H, Hasegawa Y. Lymph node density predicts lung metastasis in oral squamous cell carcinoma. Br J Oral Maxillofac Surg. 2016; 54:213-218.

7. Mansour J, Sagiv D, Alon E, Talmi Y. Pronogstic value of lymph node ratio in metastatic papillary thyroid carcinoma. J Laryngol Otol. 2018; 132:8-13.

8. Lee CW, Roh JL, Gong G, Cho KJ, Choi SH, Nam SY, Kim SY. Risk factor for recurrence of papillary thyroid carcinoma with clinically node-positive lateral neck. Ann Surg Oncol. 2015; 22:117-124.

9. Wang LY, Palmer FL, Nixon IJ, Tuttle RM, Shah JP, Patel SG, Shaha AR, Ganly I. Lateral Neck Lymph Node Characteristics Prognostic of Outcomes in Patients With Clinically Evident N1b Papillary Thyroid Cancer. Ann Surg Oncol. 2015; 22:3530-3536.
10. Hasegawa Y, Saikawa M. Update on the classification and nomenclature system for neck dissection: revisions proposed by the Japan Neck Dissection Study Group. Int J Clin Oncol. 2010; 15:5-12.

11. Takami H, Ito Y, Okamoto T, Yoshida A. Therapeutic strategy for differentiated thyroid carcinoma in Japan based on a newly established guideline managed by Japanese Society of Thyroid Surgeons and Japanese Association of Endocrine Surgeons. World J Surg. 2011; 35:111-121.

12. Amin MB, Edge S, Greene F, Byrd DR, Brookland RK, Washington MK, Gershenwald JE, Compton CC, Hess KR, Sullivan DC, Jessup JM, Brierley JD, Gaspar LE, et al. AJCC Cancer Staging Manual, 2016 8th edn. Springer, New York.

13. Brierley J, Gospodarowicz MD, Wittekind C. TNM classification of malignant tumors. International union against cancer. 8th ed. Oxford, England: Wiley-Blackwell; 2016.

14. Pak K, Cheon GJ, Nam HY, Kim SJ, Kang KW, Chung JK, Kim EE, Lee DS. Prognostic value of metabolic tumor value and total lesion glycolysis in head and neck cancer: a systematic review and meta-analysis. J Nucl Med. 2014; 55:884-90.

15. Peters E, Anzeneder T, Jackisch C, Dimpfl T, Kunz G, Katalinic A, Waldmann A. The Treatment of Primary Breast Cancer in Older Women With Adjuvant Therapy: A Retrospective Analysis of Data From Over 3000 Patients From the PATH Biobank, With Two-Year Follow-up. Dtsch Arztebl Int. 2015; 112:577-84. 